\title{
Polymerization Reactivity and Chiroptical Property of $N$-Maleoyl-L-phenylalanine Cyclohexyl Ester
}

\author{
Kensoh Kagawa and Tsutomu Oishi* \\ Department of Applied Chemistry and Chemical Engineering, \\ Faculty of Engineering, Yamaguchi University, \\ 2557 Tokiwadai, Ube, Yamaguchi 755, Japan
}

(Received October 31, 1994)

\begin{abstract}
A new type of optically active $N$-maleoyl-L-phenylalanine cyclohexyl ester (CHPAM) was synthesized from maleic anhydride, L-phenylalanine, and cyclohexanol. Radical and anionic polymerizations were carried out in several solvents at $60^{\circ} \mathrm{C}$ and $0^{\circ} \mathrm{C}$, respectively, to obtain optically active polymers. Number average molecular weights $\left(\bar{M}_{n}\right)$ of the homopolymers were $4.4 \times 10^{3}$ to $1.5 \times 10^{4}$. Specific optical rotations $\left([\alpha]_{\mathrm{D}}\right)$ of the homopolymer were -69.7 to $-65.9^{\circ}$. Radical copolymerizations of CHPAM $\left(\mathrm{M}_{1}\right)$ were performed with styrene $\left(\mathrm{ST}, \mathrm{M}_{2}\right)$ or methyl methacrylate $\left(\mathrm{MMA}, \mathrm{M}_{2}\right)$ in benzene at $60^{\circ} \mathrm{C}$. The monomer reactivity ratios $\left(r_{1}, r_{2}\right)$ and Alfrey-Price $Q-e$ were determined as follows: $r_{1}=0.27, r_{2}=0.11, Q_{1}=2.00, e_{1}=1.07$ in the CHPAM-ST system; $r_{1}=0.07, r_{2}=3.22, Q_{1}=0.38, e_{1}=1.63$ in the CHPAM-MMA system. Circular dichroism (CD) spectra of the copolymers remarkably varied with increasing content of CHPAM. Relationships between $[\alpha]_{\mathrm{D}}$ and content (wt \%) of CHPAM in the copolymer showed significant deviation from linearity. This suggests that asymmetric perturbation of the side chain chromophore and asymmetric induction in $\mathbf{M}_{1}-\mathbf{M}_{2}$ unit of the copolymer may occur.
\end{abstract}

KEY WORDS Asymmetric Induction / Asymmetric Perturbation / NSubstituted Maleimide / Styrene / Methyl Methacrylate / L-Phenylalanine Ester / Optically Active Polymer /

We reported polymerizations of $N$-substituted maleimide (RMI) bearing optically active $N$-substituents. ${ }^{1-7}$ The polymers obtained showed interesting chiroptical properties which supported asymmetric induction in the polymer main chain and asymmetric perturbation of side chain chromophores. Since RMIs have pentagonal plane structure containing a polymerizable double bond, the polymer obtained would have stereogenic centers, $(S, S)$ or $(R, R)$ in the polymer backbone ((3) in Chart 1). If one is produced more than the other, that is, if asymmetric induction occurs, the polymer main chain can be chiral and the polymer can be optically active. In addition, if the polymer is optically active, asymmetric perturbation of side chain chromophores could occur because

\footnotetext{
* To whom all correspondence should be addressed.
}

of an imide chromophore in RMI.

We reported polymerizations and copolymerizations of RMI bearing an L-amino acid residue such as L-phenylalanine (PAMI) or L-alanine (AMI). ${ }^{8}$ Chiroptical properties of the polymers obtained were clarified as well as polymerizabilities of PAMI and AMI. These RMI polymers showed specific optical rotations $\left([\alpha]_{\mathrm{D}}\right)$ and circular dichroism (CD) different from those of a model compound for each homopolymer. We observed similar results for chiroptical properties of RMI polymers bearing an optically active $\alpha$-methylbenzyl group. ${ }^{1,5,9}$ The results may indicate that the distance between a chiral carbon in $N$-substituent and maleimide ring influences the chiroptical properties of the polymer. That is, 


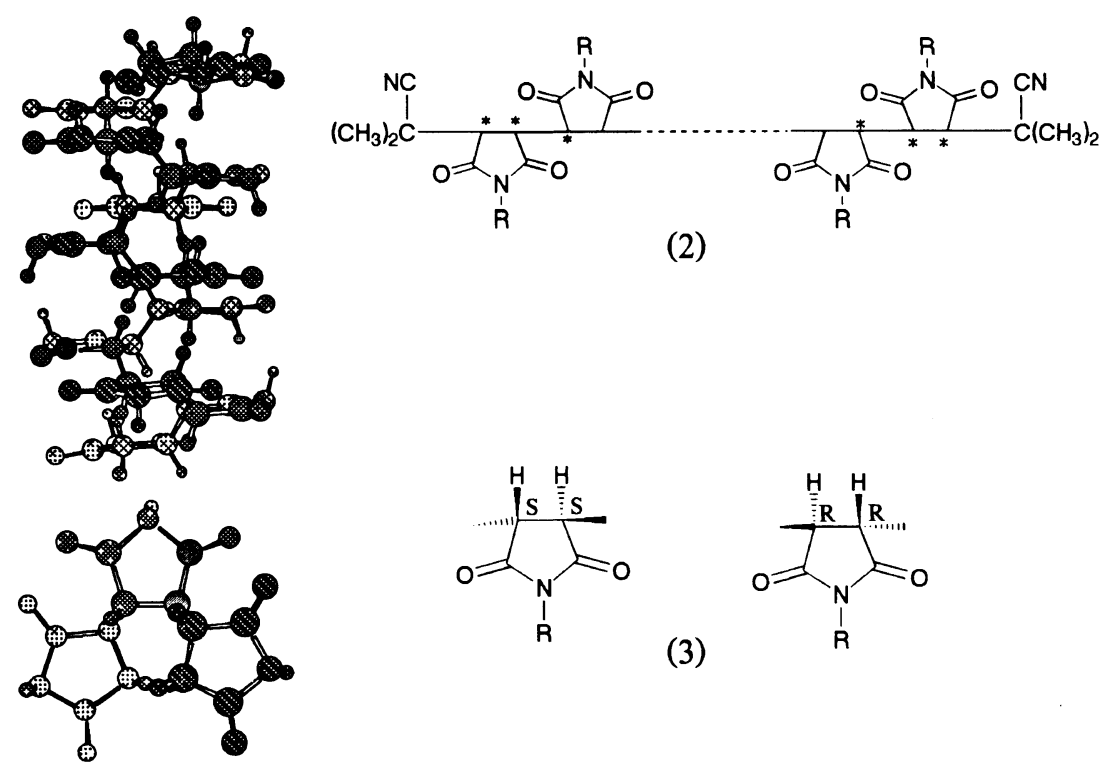

(1)

Chart 1.

the shorter the distance, the larger is the influence. PAMI and AMI polymers were thus expected to increase asymmetric induction during their polymerization and to perturb chirally the chiroptical properties of the polymer obtained. However, PAMI or AMI polymers had too low molecular weights and too wide polydispersities to study chiroptical properties of optically active polymers. Thus, by converting a carboxyl group of $N$-substituent into an ester, PAMI and $\mathrm{A} \dot{M} I$ derivatives may have higher polymerization reactivity and molecular weight of polymers obtained may be higher. Furthermore, anionic polymerizations as well as radical polymerization can be performed. We can get more detailed information about chiroptical properties of poly(RMI) containing an L-amino acid and understand the influence of a chiral carbon in RMI on asymmetric induction and perturbation.

We reported asymmetric polymerization of eleven achiral $N$-alkyl maleimides with $n$ butyllithium $/(-)$-sparteine and that all the resulting polymers were optically active. ${ }^{10}$ $N$-cyclohexyl maleimide polymer showed $[\alpha]_{\mathrm{D}}$ and Cotton effect greater than other RMI polymers. This suggests that asymmetric polymerization are affected by the bulkiness of a cyclohexyl group.

In the present work, we synthesized optically active $N$-maleoyl-L-phenylalanine cyclohexyl ester (CHPAM). CHPAM has a chiral carbon neighboring the maleimide ring and the bulkiness of a cyclohexyl group. Therefore, asymmetric induction in the polymer main chain and asymmetric perturbation of side chain chromophore may increase. CHPAM was polymerized using radical or anionic initiators and copolymerized with styrene (ST) or methyl methacrylate (MMA). The monomer reactivity ratios and Alfrey-Price $Q-e$ of CHPAM were determined. We investigated asymmetric induction in the polymer main chain and asymmetric perturbation of side chain chromophores by comparing $[\alpha]_{D}$ and CD spectra of the polymers with those of the model compound of the homopolymer. 


\section{EXPERIMENTAL}

\section{CHPAM}

CHPAM was synthesized from maleic anhydride, L-phenylalanine and cyclohexanol, as shown in Scheme 1.

$N$-Maleoyl-L-phenylalanine (PAMI). PAMI was synthesized from maleic anhydride and L-phenylalanine, as shown in the previous paper. $^{8}$

$N$-Maleoyl-L-phenylalanine Cyclohexyl Ester (CHPAM). A mixture of PAMI (12.3 g, 0.05 mol), cyclohexanol $(14.0 \mathrm{~g}, 0.14 \mathrm{~mol})$, and $p$ toluene sulfonic acid ( $p$ Ts) $(2.0 \mathrm{~g})$ in benzene $(100 \mathrm{ml})$ solution was refluxed for $12 \mathrm{~h}$. After cooling to room temperature, the benzene solution was washed with sodium hydrocarboxide in aqueous solution and water, dried on anhydrous magnesium sulfate, and evaporated to obtain crude CHPAM. The concentrate was recrystallized twice from ethanol: Yield $65 \%$ (based on PAMI); mp $60-61{ }^{\circ} \mathrm{C}$; $[\alpha]_{\mathrm{D}}=-86.0^{\circ} \quad\left(c=1 \mathrm{~g} \mathrm{dl}^{-1}, l=10 \mathrm{~cm}, \mathrm{THF}\right) ;$ ${ }^{1} \mathrm{H}$ NMR ( $\delta$ in ppm from TMS in $\mathrm{CDCl}_{3}$ ): $7.26-7.11(\mathrm{~m}, 5 \mathrm{H}$ in phenyl group), $6.59(\mathrm{~s}$, $2 \mathrm{H}, \mathrm{CH}=\mathrm{CH}), 4.96-3.41(\mathrm{~m}, 2 \mathrm{H}, \mathrm{COO}-\mathrm{CH}$ and $\mathrm{C} * \mathrm{H}), 3.54-3.36\left(\mathrm{~m}, 2 \mathrm{H}, \mathrm{CH}_{2}\right.$ connecting phenyl group), $1.82-1.25$ ( $\mathrm{m}, 10 \mathrm{H}$ in cyclohexyl group).

Model Compound of Poly(CHPAM): NSuccinoyl-L-phenylalanine Cyclohexyl Ester (CHPAS). CHPAS was prepared from succinic anhydride, L-phenylalanine and cyclohexanol according to a method similar to that of CHPAM: Yield $63 \%$ (for esterification); $\mathrm{mp}$ $84-85^{\circ} \mathrm{C} ; \quad[\alpha]_{\mathrm{D}}=-106.8^{\circ} \quad\left(c=1 \mathrm{~g} \mathrm{dl}^{-1}, \quad l=\right.$

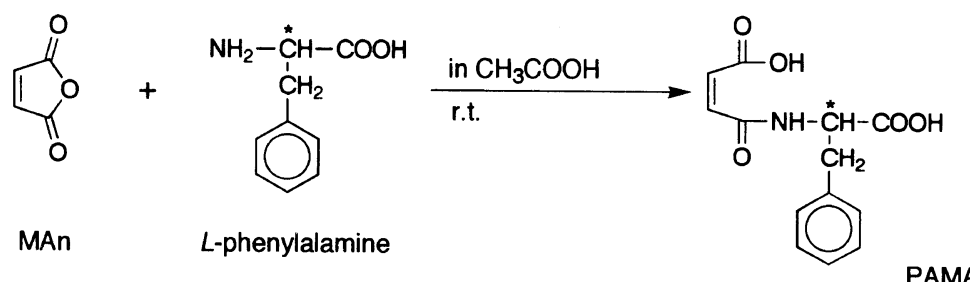<smiles>CC(C)(C)N1C(=O)CC(Cc2ccccc2)C(C(=O)O)N1C(=O)O</smiles>

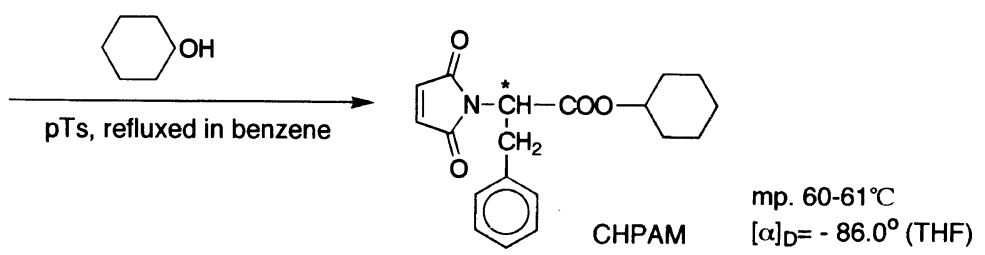

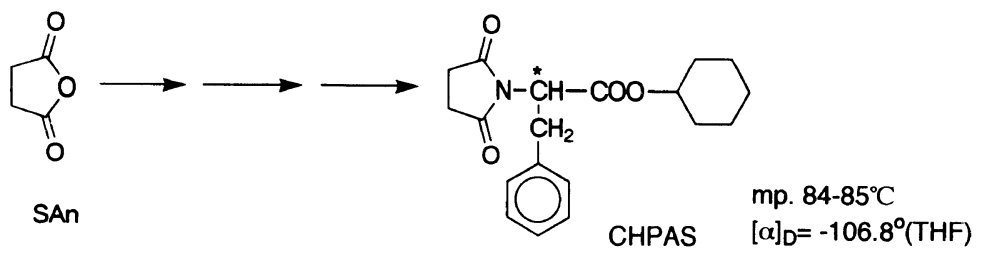

Scheme 1. 
$10 \mathrm{~cm}, \mathrm{THF}) ;{ }^{1} \mathrm{H}$ NMR $(\delta$ in ppm from TMS in $\left.\mathrm{CDCl}_{3}\right): 7.28-7.12(\mathrm{~m}, 5 \mathrm{H}$ in phenyl group), $5.02-4.96(\mathrm{~m}, 1 \mathrm{H}, \mathrm{COO}-\mathrm{CH}), 4.92-4.83(\mathrm{~m}$, $1 \mathrm{H}, \mathrm{C} * \mathrm{H}), 3.53-3.36\left(\mathrm{~m}, 2 \mathrm{H}, \mathrm{CH}_{2}\right.$ connecting phenyl group), $2.68-2.42(\mathrm{~m}, 4 \mathrm{H}$ in succinimide ring), $1.84-1.23(\mathrm{~m}, 10 \mathrm{H}$ in cyclohexyl group).

\section{Other Materials}

ST and MMA were purified by ordinary methods. ${ }^{11}$ Benzene, toluene and dioxane were purified by the usual methods ${ }^{11}$ just before use. 2,2'-Azobis(isobutyronitrile)(AIBN) was recrystallized from methanol. Commercially obtained $n$-butyllithium (BuLi) in $n$-hexane solution was used without further purification.

\section{Homopolymerization and Copolymerization}

Radical homopolymerization and copolymerization were performed with AIBN as a radical initiator in a sealed tube at $60^{\circ} \mathrm{C}$. After polymerization, the polymer solution was poured into excess methanol to precipitate the polymer. The polymer was filtered, purified by reprecipitation twice from THF solution to excess methanol, and dried in vacuo for two days. The compositions of the copolymers were determined by ${ }^{1} \mathrm{H}$ NMR spectra and nitrogen analysis data. Anionic polymerization was performed with $\mathrm{BuLi}$ as an anionic initiator in
Shlenk's reaction tube at $0^{\circ} \mathrm{C}$. After polymerization, the polymer solution was poured into excess methanol containing a few drops of dilute hydrochloric acid to precipitate the polymer. The polymer was treated by the same method as that of radical polymerization.

\section{Measurements}

D-line specific optical rotations were measured at $25^{\circ} \mathrm{C}$ in THF on a JASCO DIP-140 (Japan Spectroscopic Co.). Circular dichroism (CD) spectra were recorded at $25^{\circ} \mathrm{C}$ in $\mathrm{THF}$ on a JASCO J-20C (Japan Spectroscopic Co.) equipped with an xenon source and a computing data processor. Cell path length was $0.2 \mathrm{~mm}$. Molecular weights of polymers were measured by gel permeation chromatography (GPC) using the same techniques as described earlier. ${ }^{12}$ NMR spectra were recorded in the presence of tetramethylsilane (TMS) as an internal standard in $\mathrm{CDCl}_{3}$ solution at $25^{\circ} \mathrm{C}$ using JNM-EX-270 (JEOL Ltd.). IR and elemental analysis were conducted using the same instruments as reported previously. ${ }^{13}$

\section{RESULTS AND DISCUSSION}

\section{Homopolymerization of CHPAM}

Radical and anionic polymerizations of CHPAM were performed under several condi-

Table I. Radical and anionic polymerizations of CHPAM

\begin{tabular}{|c|c|c|c|c|c|c|c|c|c|}
\hline \multirow{2}{*}{ Run } & \multirow{2}{*}{$\frac{\text { CHPAM }}{\mathrm{moll}^{-1}}$} & \multirow{2}{*}{$\frac{\text { Initiator }}{\times 10^{-2} \mathrm{moll}^{-1}}$} & \multirow{2}{*}{$\frac{\begin{array}{c}\text { Polymn. } \\
\text { solvent }^{\mathrm{a}}\end{array}}{\mathrm{ml}}$} & \multirow{2}{*}{$\frac{\begin{array}{c}\text { Polymn. } \\
\text { temp }\end{array}}{{ }^{\circ} \mathrm{C}}$} & \multirow{2}{*}{$\frac{\begin{array}{c}\text { Polymn. } \\
\text { time }\end{array}}{h}$} & \multirow{2}{*}{$\frac{\text { Convn. }^{\mathrm{d}}}{\%}$} & \multirow{2}{*}{$\frac{\bar{M}_{n}^{\mathrm{b}}}{\times 10^{-3}}$} & \multirow{2}{*}{$\bar{M}_{w} / \bar{M}_{n}^{\mathrm{b}}$} & \multirow{2}{*}{$\frac{[\alpha]_{D}{ }^{c}}{\operatorname{deg} .}$} \\
\hline & & & & & & & & & \\
\hline $1-1$ & 0.77 & $\operatorname{AIBN}(1.0)$ & $\operatorname{BEN}(2)$ & 60 & 24 & 74.8 & 8.4 & 4.8 & -66.6 \\
\hline $1-2$ & 0.77 & $\operatorname{AIBN}(2.0)$ & BEN(2) & 60 & 24 & 86.6 & 6.7 & 6.6 & -69.7 \\
\hline $1-3$ & 0.77 & $\operatorname{AIBN}(1.0)$ & TOL(2) & 60 & 26 & 75.5 & 4.8 & 3.5 & -68.4 \\
\hline $1-4$ & 0.77 & AIBN(1.0) & TOL(2) & 60 & 26 & 75.9 & 5.9 & 1.7 & -69.9 \\
\hline $1-5$ & 0.77 & $\operatorname{AIBN}(1.0)$ & $\operatorname{DOX}(2)$ & 60 & 26 & 51.6 & 4.0 & 1.3 & -65.9 \\
\hline $1-6$ & 0.77 & $\operatorname{AIBN}(2.0)$ & $\operatorname{DOX}(2)$ & 60 & 26 & 54.9 & 3.8 & 2.9 & -66.8 \\
\hline $1-7$ & 0.77 & $\operatorname{BuLi}(3.8)$ & THF(2) & 0 & 24 & 74.2 & 5.6 & 5.6 & -133.2 \\
\hline $1-8$ & 0.61 & $\operatorname{BuLi}(3.1)$ & TOL(2.5) & 0 & 24 & 59.7 & 1.4 & 3.0 & -65.2 \\
\hline
\end{tabular}

${ }^{a}$ BEN, benzene; TOL, toluene; DOX, dioxane; THF, tetrahydrofuran. Polymn., polymerization. ${ }^{b}$ By GPC. ${ }^{\mathrm{c}} c=$ $1.0 \mathrm{~g} \mathrm{dl}^{-1}, l=10 \mathrm{~cm}$, THF. ${ }^{\mathrm{d}}$ Convn., conversion. 
tions. The results are summarized in Table I. All polymerizations proceeded homogeneously throughout except for anionic polymerization in toluene (run 1-8). In anionic polymerization in toluene, a part of the polymer precipitated. But all the polymers were soluble in chloroform and THF, thus there was no problem in NMR, GPC, $[\alpha]_{\mathrm{D}}$ and CD measurements. The polymers were white powders and showed negative optical rotations in THF at sodium $D$-line. $[\alpha]_{D}$ of the polymers were -69.7 to $-65.2^{\circ}$ except for the polymer obtained from the anionic polymerization.

Figure 1 shows the results of GPC measurements. Generally, the polymers obtained with a radical initiator had larger molecular weights than those obtained with the anionic initiator. The GPC pattern of poly(CHPAM) obtained with $\mathrm{BuLi}$ in toluene (run 1-8) overlapped two broad peaks, as shown in Figure 1, possibly since the anionic polymerization in toluene proceeded heterogeneously. The molecular weight and polydispersity of poly(CHPAM) were higher and narrower than those of poly(PAMI) reported previously. ${ }^{8}$ That is, the polymerizability of the PAMI derivative increased by converting a carboxyl group into an ester, as expected. Explanation for this may be that exchanging a carboxyl group for an ester decreases the possibility of chain transfer.

\section{Chiroptical Properties of Homopolymers}

Figure 2 shows typical CD spectra of poly(CHPAM). The polymer obtained with AIBN in benzene (run 1-1) exhibited a sharp positive and small negative Cotton effects at $225 \mathrm{~nm}$ and $260 \mathrm{~nm}$, respectively. These absorptions were ascribed to the $n \rightarrow \pi^{*}$ electronic transition of carbonyl groups and the $\pi \rightarrow \pi^{*}$ electronic transition of phenyl group. Other polymers obtained with AIBN (run 1-2 to 1-6) showed similar CD patterns. These CD patterns were almost the same as that of the model compound of CHPAM homopolymer (CHPAS), which had neither asymmetric induction nor asymmetric perturbation. But

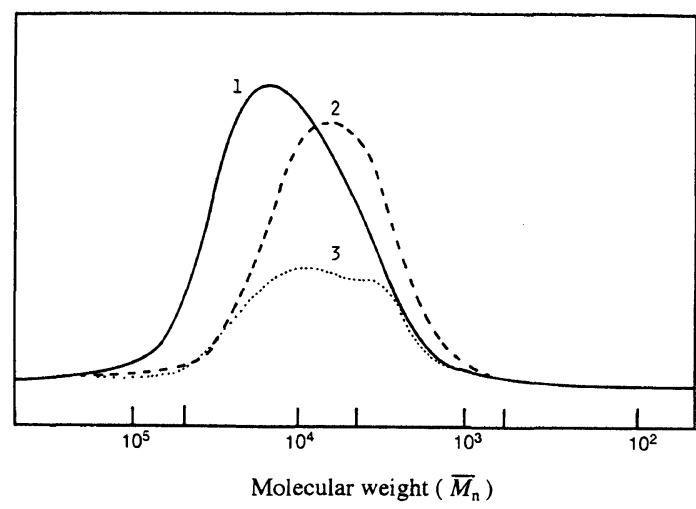

Figure 1. GPC curves for (1) poly(CHPAM) obtained with AIBN (run 1-1), (2) poly(CHPAM) obtained with BuLi in THF (run 1-7), and (3) poly(CHPAM) obtained with BuLi in toluene (run 1-8).

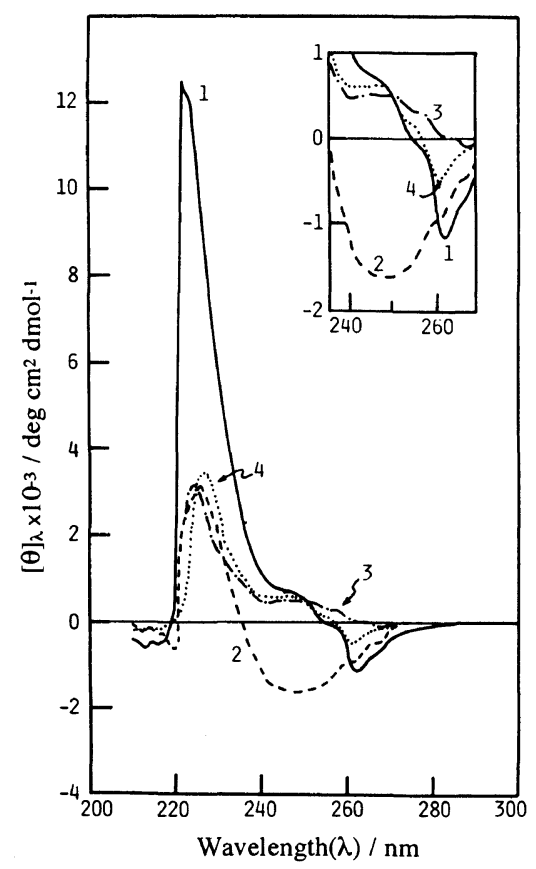

Figure 2. CD spectra for poly(CHPAM): (1) obtained with AIBN (run 1-1), (2) obtained with BuLi in THF (run 1-7), (3) obtained with BuLi in toluene (run 1-8), and (4) CHPAS

the magnitude of positive and negative Cotton effects of these polymers were about three times that of CHPAS. This may be attributable to asymmetric perturbation of side chain chromophore. 
The polymer obtained with $\mathrm{BuLi}$ in toluene (run 1-8) showed similar CD spectra to that of CHPAS. The polymer had a small molecular weight $\left(\bar{M}_{n}=1.4 \times 10^{3}\right)$. Asymmetric induction and asymmetric perturbation may thus hardly occur. The polymer obtained with BuLi in THF (run 1-7) had a similar molecular weight to that of the polymer obtained with AIBN. This polymer showed a large broad negative CD peak at around $250 \mathrm{~nm}$, and the CD pattern was quite different from that of the polymers obtained with AIBN. This indicates that the structure of the polymer main chain may differ from that of the polymer obtained with AIBN.

Figure 3 shows ${ }^{13} \mathrm{C}$ NMR spectra of the homopolymers. The signals (1 to 13 in Figure 3) of the polymer obtained with BuLi ((A) and (B) in Figure 3) were sharper than those of the polymer obtained with AIBN ((C) in Figure $3)$. This suggests that the main chain structures of the polymer obtained by anionic polymerization were more regular than those of the polymer obtained by radical polymerization. Figure 4 shows expanded ${ }^{13} \mathrm{C}-\mathrm{NMR}$ spectra of the polymers. The polymer obtained with $\mathrm{BuLi}$ in toluene showed signals at $174.8 \mathrm{ppm}$, which were not observed in others. These signals may be assigned to carbonyl carbons in the maleimide ring for some polymers having low molecular weights ((A) in Figure 4). The signals due to the main chain carbon of the polymer obtained with AIBN were broad from 47 to $40 \mathrm{pm}$. But that of the polymer obtained with BuLi split, as seen from Figure 4. This indicates that the main chains of the polymer obtained with $\mathrm{BuLi}$ had different stereo regularity from that of the polymer obtained with AIBN. Therefore, on anionic polymerizations, threo-diisotactic addition may take place to give asymmetric induction in the polymer backbone as shown in Chart 1(3). One of the split signal could be assigned to main chain carbons bearing the $(S, S)$ or $(R, R)$ structure (i.e., trans), and the other the $(R, S)$ or $(S, R)$ structure (i.e., cis), according to the assignment of poly( $N$-phenylmaleimide) reported by But- ler et al. ${ }^{14}$ Though we can not determine which signal ascribed to trans or cis structure in our present work, the difference of CD pattern of the polymer (run 1-7) was attributable to the predominant structure of $(S, S)$ or $(R, R)$ in the main chain. As shown in Figure 4 (A), the split signals were observed at 47 to $40 \mathrm{ppm}$ for the polymer obtained with BuLi in toluene. But the CD spectra of this polymer differed from those of the polymer obtained in THF, and were rather similar to those of CHPAS. The molecular weight of the polymer (run 1-8) was so small $\left(\bar{M}_{n}=1.4 \times 10^{3}\right)$ that asymmetric induction and asymmetric perturbation could not occur. This suggests that asymmetric induction and asymmetric perturbation require to some extent a high polymer molecular weight.

We propose three models as asymmetric induction in RMI polymer main chain in Chart 1: (1) a helix of RMI polymer, (2) chiral carbons occur in the end group, and (3) displacement of stereogenisity in the maleimide ring. Though we can not determine the main chain structure, (1) was hardly incorporated to the main chain structure. This can be judged from NMR study. (2) may scarcely influence chiroptical properties of polymers, as shown by the chiroptical properties of the polymer obtained with BuLi in toluene. This polymer had more end groups than others because of its smaller molecular weight $\left(\bar{M}_{n}=1.4 \times 10^{3}\right)$. Thus, it is expected that the induction (2) influences chiroptical properties. However $[\alpha]_{D}$ and CD spectra of the polymer were similar to those of CHPAS. This suggests that asymmetric induction (2) hardly affects chiroptical properties.

On the other hand, we have to consider asymmetric perturbation ${ }^{15}$ for all obtained polymers because CHPAM polymer had a chiral carbon, a phenyl group, and carbonyl groups which can cause asymmetric perturbation. The main chain of the polymers obtained with AIBN had poorer stereo regularity than that obtained with BuLi judging from ${ }^{13} \mathrm{C}$ NMR spectra (Figures 3 and 4). If the main 
(A)

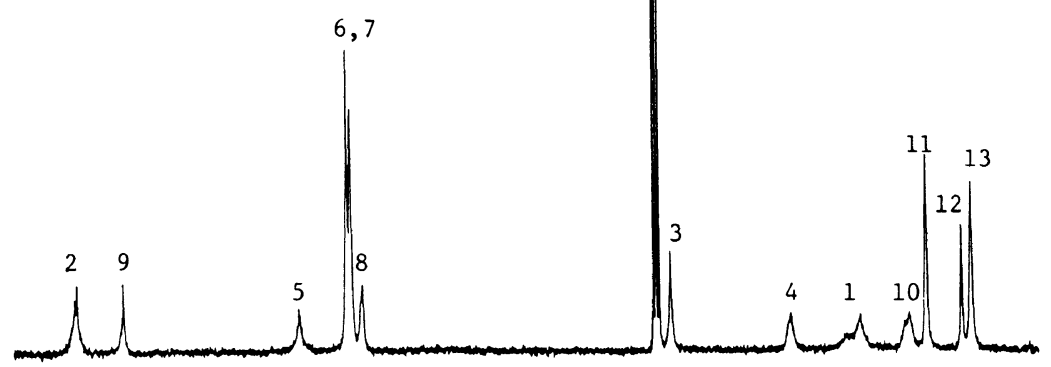

(B)

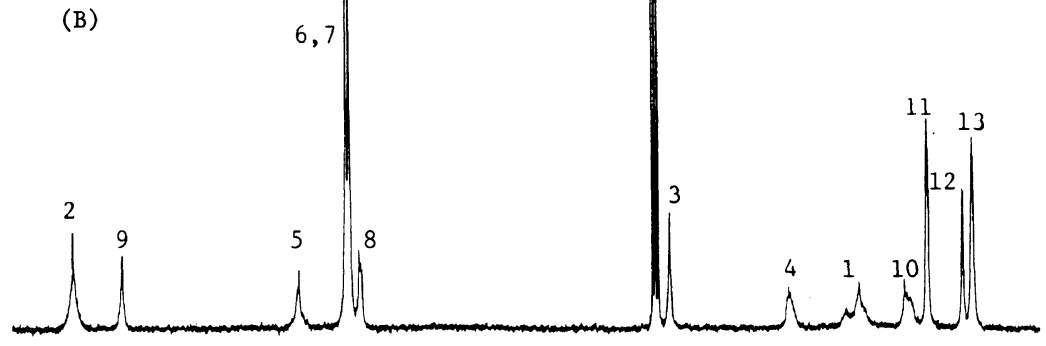

(c)

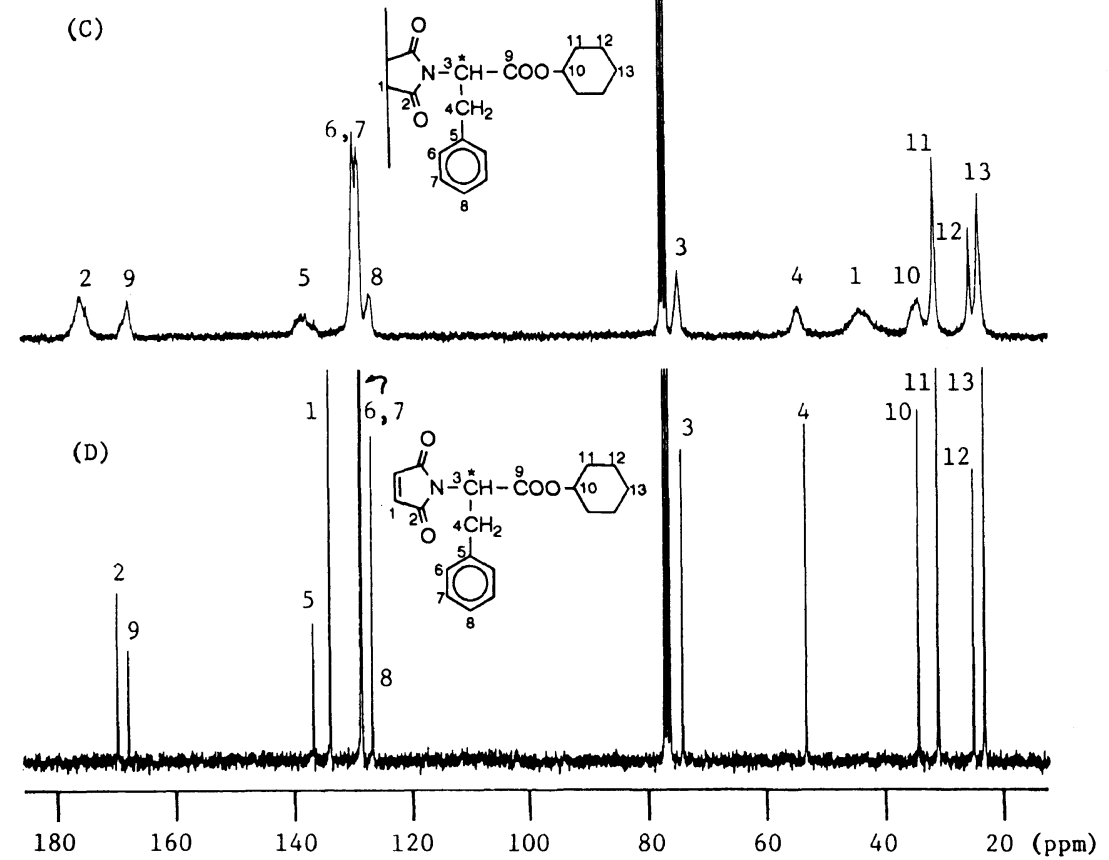

Figure 3. ${ }^{13} \mathrm{C}$ NMR spectra for poly(CHPAM) and CHPAM monomer: (A) polymer obtained with BuLi in toluene (run 1-8), (B) polymer obtained with BuLi in THF (run 1-7), (C) polymer obtained with AIBN in benzene (run 1-1), and (D) CHPAM monomer. 


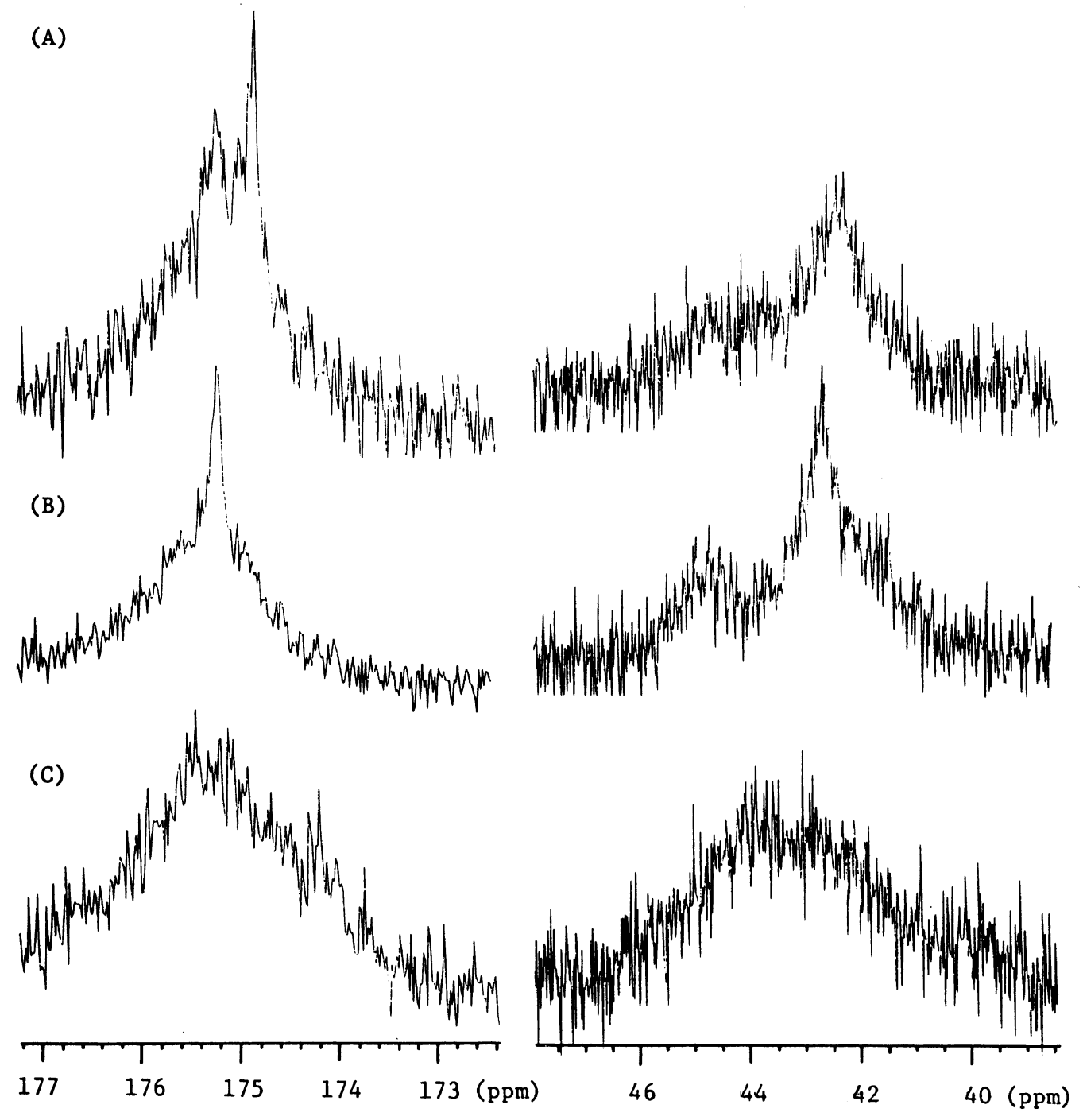

Figure 4. Expanded ${ }^{13} \mathrm{C}$ NMR spectra for poly(CHPAM): (A) obtained with BuLi in toluene (run 1-8), (B) obtained with BuLi in THF (run 1-7), and (C) obtained with AIBN in benzene (run 1-1)

chain of the polymer had stereo regularity such as the polymer obtained with $\mathrm{BuLi}$ (run 1-7 and 8), the CD pattern of the polymer could differ from that of CHPAS. But the CD pattern of the polymers obtained with AIBN was very similar to that of CHPAS as shown in Figure 2. Accordingly, the chiroptical properties of the polymer obtained with AIBN were mainly characterized by asymmetric perturbation of side chain chromophores. In the present study, it was difficult to determine to what extent asymmetric perturbation affects chiroptical properties of the polymer. This is because the complete removal of the optically active moiety was unsuccessful using acid and base catalysts, such as $\mathrm{HCl}, \mathrm{H}_{2} \mathrm{SO}_{4}, \mathrm{HBr}, \mathrm{NaOH}, \mathrm{KOH}$, and hydrazine monohydrate, and phenylhydrazine. ${ }^{1,5,9}$ However, these results support that a chiral carbon neighboring the maleimide ring influences the chiroptical properties of the 
Table II. Radical copolymerization of $\operatorname{CHPAM}\left(\mathrm{M}_{1}\right)$ with $\mathrm{ST}\left(\mathrm{M}_{2}\right)$ or MMA( $\left(\mathrm{M}_{2}\right)$ in benzene $(2 \mathrm{ml})$ at $60 \mathrm{OCa}$

\begin{tabular}{|c|c|c|c|c|c|c|c|c|}
\hline \multirow{2}{*}{ Run } & \multirow{2}{*}{$M_{2}$} & \multirow{2}{*}{$\begin{array}{c}\begin{array}{c}M_{1} \text { in } \\
\text { monomer }\end{array} \\
\text { mol\% }\end{array}$} & \multirow{2}{*}{$\begin{array}{c}\begin{array}{c}\text { Polymn. } \\
\text { time }\end{array} \\
\mathrm{h}\end{array}$} & \multirow{2}{*}{$\frac{\text { Convn. }}{\%}$} & \multirow{2}{*}{$\begin{array}{c}\begin{array}{c}\mathrm{M}_{1} \text { in } \\
\text { copolymer }\end{array} \\
\mathrm{mol} \%\end{array}$} & \multirow{2}{*}{$\frac{\bar{M}_{n}^{\mathrm{b}}}{\times 10^{-4}}$} & \multirow{2}{*}{$\bar{M}_{w} / \bar{M}_{n}^{\mathrm{b}}$} & \multirow{2}{*}{$\frac{[\alpha]_{D}{ }^{c}}{\operatorname{deg} .}$} \\
\hline & & & & & & & & \\
\hline $2-1$ & ST & 5.1 & 6 & 27.9 & 18.8 & 2.70 & 3.00 & -57.6 \\
\hline $2-2$ & ST & 9.8 & 6 & 33.2 & 24.0 & 2.34 & 4.86 & -74.7 \\
\hline $2-3$ & ST & 17.9 & 6 & 39.9 & 40.4 & 6.73 & 2.60 & -85.2 \\
\hline $2-4$ & ST & 29.7 & 6 & 32.9 & 47.6 & 11.1 & 2.44 & -92.2 \\
\hline $2-5$ & ST & 49.4 & 6 & 39.1 & 51.2 & 12.6 & 2.39 & -96.7 \\
\hline $2-6$ & ST & 69.3 & 6 & 30.0 & 55.2 & 11.4 & 2.56 & -99.4 \\
\hline $2-7$ & ST & 89.5 & 6 & 39.3 & 84.5 & 0.46 & 13.9 & -78.3 \\
\hline $2-8$ & MMA & 5.0 & 12 & 47.8 & 2.0 & 4.61 & 1.67 & -8.4 \\
\hline $2-9$ & MMA & 10.0 & 9 & 43.1 & 4.3 & 4.40 & 2.18 & -16.0 \\
\hline $2-10$ & MMA & 21.5 & 12 & 34.8 & 7.9 & 4.05 & 2.17 & -33.3 \\
\hline $2-11$ & MMA & 30.0 & 12 & 34.3 & 15.1 & 4.41 & 1.95 & -47.1 \\
\hline $2-12$ & MMA & 49.9 & 12 & 40.9 & 21.2 & 3.48 & 2.26 & -70.6 \\
\hline $2-13$ & MMA & 69.9 & 6 & 26.3 & 49.0 & 1.99 & 3.12 & -78.0 \\
\hline $2-14$ & MMA & 89.3 & 6 & 27.8 & 75.5 & 1.73 & 2.80 & -66.9 \\
\hline
\end{tabular}

${ }^{\mathrm{a}}[\mathrm{AIBN}]=1.0 \times 10^{-2} \mathrm{moll}^{-1} ; \mathrm{M}_{1}+\mathrm{M}_{2}=1.0 \mathrm{~g} .{ }^{\mathrm{b}} \mathrm{By} \mathrm{GPC} .{ }^{\mathrm{c}} c=1.0 \mathrm{~g} \mathrm{dl}^{-1} ; l=10 \mathrm{~cm} ; \mathrm{THF}$.

polymer.

\section{Radical Copolymerizations of CHPAM with $S T$ and $M M A$}

Radical copolymerizations of CHPAM( $\left.\mathrm{M}_{1}\right)$ with $\operatorname{ST}\left(M_{2}\right)$ and $\operatorname{MMA}\left(M_{2}\right)$ were performed in benzene at $60^{\circ} \mathrm{C}$ in the presence of AIBN. The results of copolymerizations are summarized in Table II. All copolymerizations proceeded homogeneously. The copolymers were white powders and optically active. $\bar{M}_{n}$ of the copolymers were greater than those of the homopolymers. Polydispersity $\left(\bar{M}_{w} / \bar{M}_{n}\right)$ of the copolymers was narrower than that of poly(PAMI-co-ST) or poly(PAMI-co-MMA) reported previously. ${ }^{8}$ The reason for this may be that CHPAM had no carboxyl groups which were liable to chain transfer reaction.

Copolymer composition curves for the copolymerizations of CHPAM $\left(\mathrm{M}_{1}\right)$ with ST $\left(\mathrm{M}_{2}\right)$ or MMA $\left(\mathrm{M}_{2}\right)$ are shown in Figure 5. Monomer reactivity ratios $\left(r_{1}, r_{2}\right)$, calculated from the high conversion method reported by Tüdös et $a l^{16}$ and Alfrey-Price $Q-e^{17}$ were determined as follows: $r_{1}=0.27, r_{2}=0.11$, $Q_{1}=2.00, e_{1}=1.07$ in the CHPAM-ST system

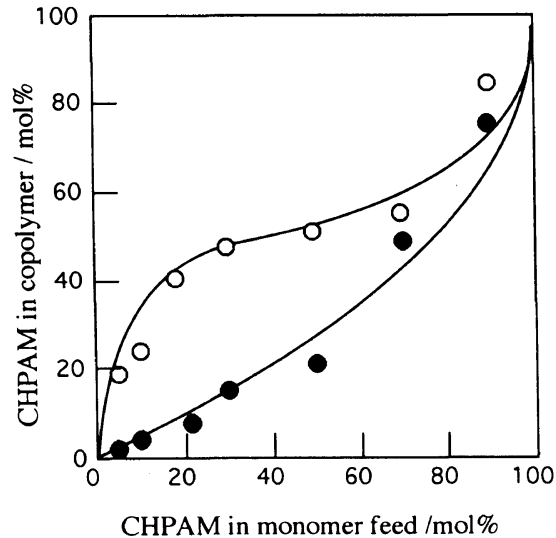

Figure 5. Copolymer composition curves for (O) CHPAM-ST system and (O) CHPAM-MMA system.

and $r_{1}=0.07, r_{2}=3.22, Q_{1}=0.38, e_{1}=1.63$ in the CHPAM-MMA system. $Q_{1}$ in the CHPAM-ST system were larger than those in the CHPAM-MMA system. This may be due to the formation of a charge transfer (CT) complex. ${ }^{18}$ These copolymerization parameters showed the same tendencies as other RMI reported previously. ${ }^{1-8}$ 


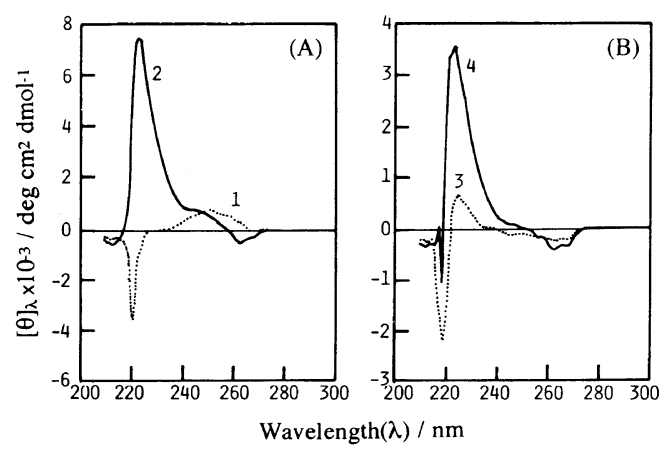

Figure 6. CD spectra for (A) poly(CHPAM-co-ST) and (B) poly(CHPAM-co-MMA); (1) $55.2 \mathrm{~mol} \%$ of CHPAM (run 2-6), (2) $84.5 \mathrm{~mol} \%$ of CHPAM (run 2-7), (3) $21.2 \mathrm{~mol} \%$ of CHPAM (run 2-12), and (4) $49.0 \mathrm{~mol} \%$ of CHPAM (run 2-13).

\section{Chiroptical Properties of Copolymers}

Figure 6 shows the CD spectra for poly(CHPAM-co-ST) and poly(CHPAM-co-MMA). Both the copolymers containing more than $c a$. $50 \mathrm{~mol} \%$ CHPAM (2 and 4 in Figure 6) showed a CD pattern similar to that of CHPAM homopolymer. That is, a positive Cotton effect at $225 \mathrm{~nm}$ and negative Cotton effects at about $260 \mathrm{~nm}$ were observed. For copolymers containing less than $c a .50 \mathrm{~mol} \%$ CHPAM, the copolymers showed quite different CD patterns from that of the homopolymer (1 and 3 in Figure 6). In our previous investigations on optically active RMI, ${ }^{1-9}$ the CD pattern did not change with the composition, but the magnitude of the Cotton effect changed with copolymer composition. In the present work, we observed that $\mathrm{CD}$ pattern of the copolymer significantly varied with copolymer composition. It is clear that $\operatorname{ST}\left(M_{2}\right)$ or $\operatorname{MMA}\left(M_{2}\right)$ units can affect the chiroptical properties of the copolymers. The structural difference between the copolymer and homopolymer was due to $M_{1}-M_{2}$ successive units or absence of the units in the polymer. Therefore, it can be considered that the CD pattern for 1 or 3 in Figure 6 is characterized by the $M_{1}-M_{2}$ successive units in the copolymer. That is, asymmetric perturbation of side chain chromophores is influenced by phenyl chromophores of $\mathrm{ST}\left(\mathrm{M}_{2}\right)$ units or

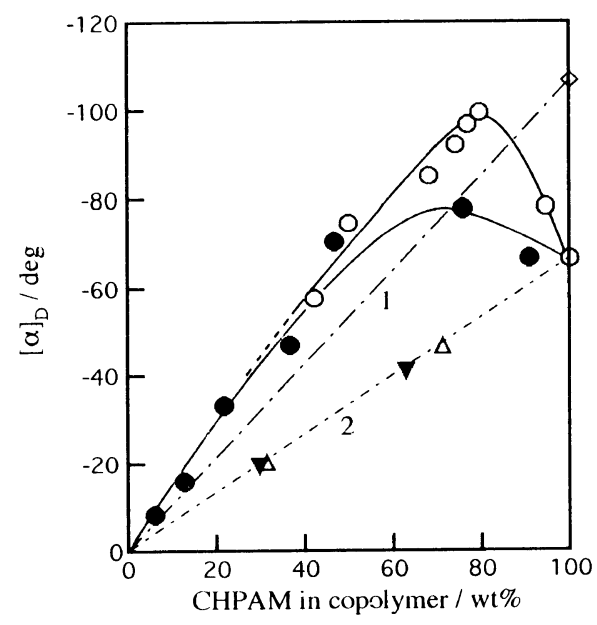

Figure 7. Relationships between specific optical rotations $\left([\alpha]_{\mathrm{D}}\right)$ and CHPAM content $(\mathrm{wt} \%)$ in a copolymer; (○) poly(CHPAM-co-ST), (O) poly(CHPAM-co-MMA), $(\triangle)$ mixture of poly $($ CHPAM) and poly $(\mathrm{ST}),(\boldsymbol{\nabla})$ mixture of poly(CHPAM) and poly(MMA), and $(\diamond)$ CHPAS.

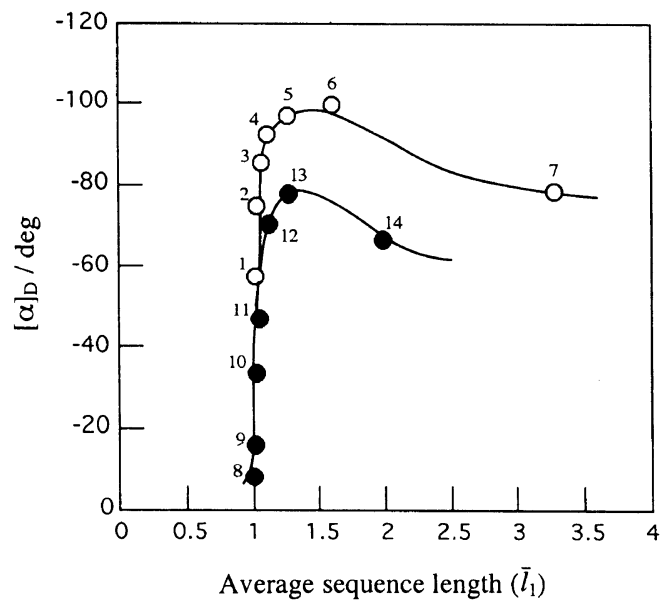

Figure 8. Relationships between specific optical rotations $\left([\alpha]_{\mathrm{D}}\right)$ and average sequence length $\left(T_{1}\right)$ of a copolymer; (O) poly(CHPAM-co-ST) and (O) poly(CHPAM-co-MMA). Numbers corresponding to run numbers in Table II.

carbonyl chromophores of $\operatorname{MMA}\left(\mathrm{M}_{2}\right)$ units, and asymmetric induction in the $\mathbf{M}_{1}-\mathbf{M}_{2}$ successive units. $^{8}$ This is supported by the results in Figures 7 and 8.

Figure 7 shows relationships between $[\alpha]_{D}$ of the copolymer and CHPAM content (wt $\%)$ 
of the copolymer. Line 1 is straight one drawn through $[\alpha]_{D}$ of CHPAS and zero. The line 1 can indicate that neither asymmetric induction nor asymmetric perturbation occurs because relationships between $[\alpha]_{\mathrm{D}}$ and CHPAS content $(\mathrm{wt} \%)$ in the mixture of CHPAS and poly(ST) or poly(MMA) agree with the line. Line 2 is straight one drawn through $[\alpha]_{D}$ of CHPAS and zero. The line 2 can indicate that neither asymmetric induction nor asymmetric perturbation occur in $\mathbf{M}_{1}-\mathbf{M}_{2}$ successive units. Deviation from line 2 increased when CHPAM was contained less than 80 or $70 \mathrm{wt} \%$ in poly(CHPAM-co-ST) or poly(CHPAM-coMMA), respectively. When the content of CHPAM was more than 80 or $70 \mathrm{wt} \%$, deviation from line 2 decreased and $[\alpha]_{D}$ of the copolymers approached that of the homopolymer. Polymers containing CHPAM less than $c a .80$ or $70 \mathrm{wt} \%$ showed quite different CD spectra from that of the homopolymer, as shown in Figure 6 (1 and 3). This suggests that asymmetric induction and asymmetric perturbation occurs in the $\mathbf{M}_{1}-\mathbf{M}_{2}$ successive units.

Figure 8 shows relationships between $[\alpha]_{\mathrm{D}}$ of the copolymer and average sequence length $\left(\bar{l}_{1}\right)^{8}$ of CHPAM in the copolymers. Poly(CHPAM-co-ST) corresponding to plots 1 to 6 in Figure 8 showed the same CD pattern as 1 in Figure 6(A). Poly(CHPAM-co-MMA) corresponding to plots 8 to 12 showed the same CD pattern as 3 in Figure 6(B). When $\bar{l}_{1}$ of these copolymers was from 1 to $1.5, \mathrm{M}_{1}-\mathrm{M}_{2}$ successive units were rich in the copolymers. But when $\bar{l}_{1}$ was more than 1.5 which corresponded to plots 7, 13, and 14 in Figure 8, $[\alpha]_{\mathrm{D}}$ and CD pattern became similar to those of the homopolymer. This suggests that $\mathbf{M}_{1}-$ $\mathbf{M}_{2}$ successive units affect the chiroptical properties of a copolymer.

Similar results such as in Figures 7 and 8 were also observed for the polymer which had a chiral carbon neighboring the maleimide ring. ${ }^{1,5,8}$ CHPAM copolymers with ST or MMA were composed of $\mathbf{M}_{1}-\mathbf{M}_{2}$ successive units similar to those of optically active RMI copolymer reported previously, ${ }^{1-9}$ judging from copolymerization parameters. Thus, poly(CHPAM-co-ST) or poly(CHPAM-co-MMA) may have chiral carbons in $\mathbf{M}_{1}-\mathbf{M}_{2}$ successive units because of a pro-chiral carbon in ST or MMA. The phenyl group in ST or carbonyl group in MMA could chirally perturb chiroptical property of the copolymers. But the results given in Figure 6 were characteristic of the CHPAM copolymer with ST or MMA. This may be attributable to bulkiness of cyclohexyl group and chromophores of phenyl or carbonyl group neighboring the chiral carbon. This suggests that the environment of the chiral carbon such as L-phenylalanine residue influences asymmetric induction and asymmetric perturbation in the $M_{1}-M_{2}$ successive units.

\section{CONCLUSION}

(1) Optically active $N$-maleoyl-L-phenylalanine cyclohexyl ester (CHPAM) was synthesized as an $\mathrm{N}$-substituted maleimide derivative bearing an amino acid residue. CHPAM was polymerized with radical or anionic initiators to give optically active polymers.

(2) Polymerizability of CHPAM was higher than that of the non-esterified-type monomer (PAMI). From the results of radical copolymerizations of CHPAM $\left(M_{1}\right)$ with $\mathrm{ST}\left(\mathrm{M}_{2}\right)$ or $\operatorname{MMA}\left(\mathrm{M}_{2}\right)$, the monomer reactivity ratios $\left(r_{1}, r_{2}\right)$ and Alfrey-Price $Q-e$ were determined as follows: $r_{1}=0.27, r_{2}=0.11 ; Q_{1}=2.00$, $e_{1}=1.07$ in the CHPAM-ST system, $r_{1}=0.07$, $r_{2}=3.22, Q_{1}=0.38, e_{1}=1.63$ in the CHPAMMMA system.

(3) From the chiroptical properties of CHPAM homopolymer, it was shown that both asymmetric induction in the polymer main chain and asymmetric perturbation of side chain chromophores occur.

(4) The CD patterns of poly(CHPAM-coST) and poly(CHPAM-co-MMA) remarkably changed with copolymer composition; chiroptical properties of the copolymers. That is, asymmetric induction and asymmetric pertur- 
bation were affected by $M_{1}-M_{2}$ successive units in the copolymer.

Acknowledgment. We are thankful to $\mathrm{Mr}$. M. Momoi for carrying out the elemental analysis.

\section{REFERENCES}

1. T. Oishi and M. Fujimoto, J. Polym. Sci., Polym. Chem. Ed., 22, 2789 (1984).

2. T. Oishi., A. Kamori, and M. Fujimoto, J. Macromol. Sci., Pure Appl. Chem., A29, 231 (1992).

3. T. Oishi and M. Fujimoto, J. Polym. Sci., A, Polym. Chem. Ed., 30, 1821 (1992).

4. T. Oishi, Y. Otsubo, and M. Fujimoto, Polym. J., 24, 527 (1992).

5. T. Oishi, K. Kagawa, and M. Fujimoto, Macromolecules, 26, 24 (1993).

6. T. Oishi, K. Kagawa, and M. Fujimoto, Polym. J., 25, 977 (1993).

7. T. Oishi, K. Kagawa, and M. Fujimoto, Polymer, 34,
2644 (1993).

8. T. Oishi, K. Matsusaki, and M. Fujimoto, Polym. J., 24, 1281 (1992).

9. T. Oishi, K. Kagawa, and M. Fujimoto, J. Polym. Sci., A. Polym. Chem., in press.

10. T. Oishi, H. Yamasaki, and M. Fujimoto, Polym. J., 23, 795 (1991).

11. T. Otsu and M. Kinoshita, "Koubunshigousei no Jikkenhou,” Kagakudojin, Kyoto, 1972, pp 69-91.

12. T. Oishi, Y. Morioka and M. Fujimoto, Polym. J., 21, 287 (1989).

13. T. Oishi and M. Fujimoto, J. Polym. Sci., Polym. Chem. Ed., 20, 2727 (1982)

14. Kurt G. Olson, and George B Butler, Macromolecules, 17, 2486 (1984).

15. G. Wulff and P. K. Dhal, Macromolecules, 23, 100 (1990).

16. F. Tödös, T. Kelen, T. Folde-Berezsnich, and B. Turcsanyi, J. Macromol. Sci.-Chem., A10, 1513 (1976).

17. T. Alfrey, Jr. and C. C. Price, J. Polym. Sci., 2, 101 (1947).

18. T. Oishi, M. Iwahara, and M. Fujimoto, Polym.J., 23, 1409 (1991). 